\title{
Formation of crystalline EVAL membranes by controlled mass transfer process in water-DMSO-EVAL copolymer systems
}

\author{
Liao-Ping Cheng ${ }^{\mathrm{a}, *}$, Tai-Horng Young ${ }^{\mathrm{b}}$, Wei-Ming You ${ }^{\mathrm{a}}$ \\ ${ }^{a}$ Department of Chemical Engineering, Tamkang University, Taipei, ROC Taiwan \\ ${ }^{\mathrm{b}}$ Center for Biomedical Engineering, College of Medicine, National Taiwan University, Taipei, ROC Taiwan
}

Received 26 September 1997; received in revised form 5 January 1998; accepted 3 February 1998

\begin{abstract}
The effect of solvent content in the coagulation bath on the formation of poly(ethylene-co-vinyl alcohol) (EVAL) membranes by isothermal immersion-precipitation in water-dimethylsulfoxide (DMSO)-EVAL system has been investigated. As a homogeneous EVAL dope is immersed in a harsh bath, e.g., water, instant precipitation occurs initiated by the liquidliquid demixing process. The formed membrane exhibits an asymmetric morphology with extensive finger-like macrovoids, similar to that observed in most amorphous polymeric membranes. On the other hand, if precipitation takes place in a soft bath containing a substantial amount of solvent, crystallization, rather than liquid-liquid demixing, starts to dominate and a skinless, bi-continuous, particulate membrane becomes the precipitated product. The immersion-precipitation process for the present system has been modeled as a ternary mass transfer problem. The calculated diffusion trajectories and concentration profiles illustrate reasonably the membrane morphologies formed in various immersion conditions. (C) 1998 Elsevier Science B.V.
\end{abstract}

Keywords: EVAL; Crystallization; Liquid-liquid demixing; Immersion-precipitation; Diffusion

\section{Introduction}

Studies on the membrane formation mechanism have long been focused upon the precipitation process of amorphous polymers from nonsolvent solutions. A number of theories have emerged since the early work of Maier and Scheuermann [1]; among which, "phase inversion" and "nucleation and growth" of liquid pores produced by phase demixing are ubiquitously considered as the causes of pore formation $[2,3]$. These concepts are, however, inadequate for interpret-

\footnotetext{
*Corresponding author.
}

ing the complex morphology of membranes made from crystallizable polymers in wet quench procedures. In the latter case, precipitation can occur both by the processes of liquid-liquid demixing and crystallization [4-7] to yield membranes exhibiting characters from both types of phase separations. Liquidliquid demixing results in various cellular structures $[2,3]$. The cellular pores can either be fully isolated or interconnected, depending upon the rigidity of the polymer-rich phase surrounding the growing micelles. In the extreme case, pores can even develop into finger-shape macrovoids. Crystallization brings particulate features onto the pore wall of the membrane [4-7]. The crystalline particles can have different 
shapes, e.g., stick, sheaf, sphere, representing different stages of crystallization [8], and the size of the particle can vary widely from a tenth of a $\mu \mathrm{m}$ to several tens of a $\mu \mathrm{m}$. Crystallization sometimes takes place extensively such that the cellular pore becomes highly distorted and the cell wall becomes diffuse. Cheng et al. $[4,5,9]$ pointed out that the sequence of precipitation events affects most significantly the relative weight of both types of phase separation on the formed membrane morphology. In general, crystallization is a slower process than liquid-liquid demixing because it requires reorientation of the polymer chain into the crystalline lattice. Therefore, under normal immersion-precipitation conditions, crystallization does not occur until liquid-liquid phase separation has completely set in the membrane structure. That is why crystallization phenomenon has often been overlooked in most membrane literature.

Poly(ethylene-co-vinyl alcohol) (EVAL) is a crystalline copolymer which can be precipitated from dimethylsulfoxide (DMSO, solvent)-water (nonsolvent) solutions to form microporous particulate membranes [10,11]. The equilibrium phase behavior of this membrane formation system has been described previously [11]. Membranes having typical cellular and particulate morphologies were also demonstrated, which were prepared by good and incipient (with respect to crystallization) dope solutions, respectively. In this work, the effect of coagulation bath on the process of EVAL precipitation will be discussed. Ordinarily, by carrying out the precipitation in a soft bath (high solvent content), liquid-liquid demixing can be effectively suppressed because, in this case, the rate of solvent-nonsolvent exchange is decreased and the binodal miscibility envelop is entered sluggishly. Polymer crystallization, thus, may occur prior to liquid-liquid demixing to form morphologies mixed with different contents of cellular pores and particles. In addition, in order to have a clear insight into the membrane formation mechanism, kinetic events encountered in the immersion-precipitation process are modeled as a ternary diffusion problem [12-16]. Computations are performed to obtain the composition distribution of each component in the membrane just before phase separation takes place. These results are found to be consistent with the light transmission measurements and the membrane morphologies prepared in different precipitation baths.

\section{Theory}

The immersion-precipitation process as shown schematically in Fig. 1 for preparing polymeric porous membranes has been described previously [12-16]. The mass transfer equations for the membrane solution region were demonstrated in different formates by Cohen et al. [12], Reuvers et al. [13], Reuvers and Smolders [14], and Tsay and McHugh [15]. Eqs. (1) and (2) below are derived by Cheng [16]:

$$
\begin{aligned}
& \frac{\partial\left(\phi_{1} / \phi_{3}\right)}{\partial \tau}=\frac{V_{1}}{D_{0}} \frac{\partial\left(\phi_{3} L_{11}\left(\partial \mu_{1} / \partial \eta\right)+\phi_{3} L_{12}\left(\partial \mu_{2} / \partial \eta\right)\right)}{\partial \eta} \\
& \frac{\partial\left(\phi_{2} / \phi_{3}\right)}{\partial \tau}=\frac{V_{2}}{D_{0}} \frac{\partial\left(\phi_{3} L_{21}\left(\partial \mu_{1} / \partial \eta\right)+\phi_{3} L_{22}\left(\partial \mu_{2} / \partial \eta\right)\right)}{\partial \eta}
\end{aligned}
$$

where $\phi_{i}$ and $V_{i}$ are the volume fraction and the partial molar volume of component $i$. In the present notation,

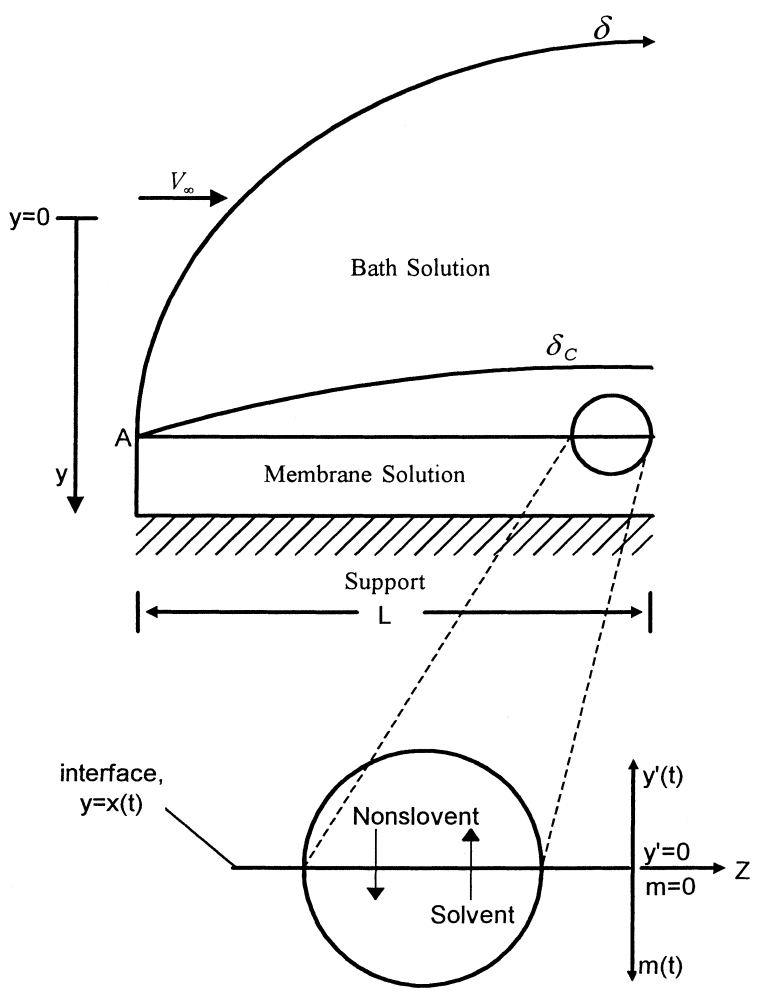

Fig. 1. Schematic representation of the immersion-precipitation process. 
components 1, 2, and 3 refer to nonsolvent, solvent, and polymer, respectively. $\mu_{i}$ in these equations are the chemical potentials of species $i(i=1$ and 2$)$. Their values are computed using a modified Flory-Huggins expression for ternary systems [16]. Eqs. (1) and (2) are transport equations expressed in dimensionless forms. The dimensionless time, $\tau$, and the dimensionless distance, $\eta$, are defined as: $\tau=D_{0} t / M^{2}$ and $\eta=m / M$, where $D_{0}$ is the pre-exponential factor for the diffusion coefficient in Duda's expression [17]; $M$ is the volume per unit area of the initial membrane solution before immersion. The polymer coordinate, $m$, as shown in Fig. 1, is defined as the volume of polymer between the membrane-bath interface and the position of observation per unit area. $L_{i j}(i, j=1,2)$ in Eqs. (1) and (2) are coefficients for the phenomenological expressions of diffusional fluxes [18]. According to Onsager's law, the cross coefficients must be equal, i.e., $L_{12}=L_{21}$ [18].

Since polymer is excluded from the bath, the equation of continuity for bath region is identical to that for a binary system and is given in dimensionless form [16]:

$$
\begin{aligned}
\frac{\partial \phi_{1}}{\partial \tau}= & \frac{M^{2}}{\delta_{c}^{2}} \frac{\partial\left(\left(D_{12} / D_{0}\right)\left(\partial \phi_{1} / \partial Y\right)\right)}{\partial Y} \\
& +\frac{1}{D_{0}}\left(-\frac{3}{8} \frac{V_{\infty} M^{2} \delta_{c} Y^{2}}{k \sqrt{z^{3}}}+\frac{3}{16} \frac{V_{\infty} M^{2} \delta_{c}^{3} Y^{4}}{k^{3} \sqrt{z^{5}}}\right) \\
& \times\left(\frac{\partial \phi_{1}}{\partial Y}\right)(f+1)
\end{aligned}
$$

The coordinate of the bath region, as shown in Fig. 1, is adopted such that the interface is always at $y^{\prime}$ equal to zero (i.e., $y^{\prime}=-y+X(t), X(t)$ is the position of the interface). In Eq. (3), the dimensionless distance, $Y$, is defined to be equal to $y^{\prime} / \delta_{c}$, where $\delta_{c}=\delta\left(D_{12} / \nu\right)^{1 / 3}$ is the thickness of the concentration boundary layer; $\delta=4.64\left(\nu z / v_{\infty}\right)^{1 / 2}$ is the thickness of the hydrodynamics boundary layer; $\nu$ and $D_{12}$ are the kinematic viscosity and the mutual diffusion coefficient for the bath liquid; $v_{\infty}$ is the velocity of the free stream; $k$ in Eq. (3) is equal to $4.64\left(\nu / V_{\infty}\right)^{0.5}$; and $f$ is a factor which accounts for the effect of mass transfer in the $z$ direction $[16,19]$. Calculated results for the case of $f=0$ are reported in this work.

The initial and boundary conditions are identical to those given in a previous publication [16]. Interfacial equilibrium is assumed to be established instantly after contact of the membrane solution with the bath.
Hence, the interfacial compositions are always located at the binodal (i.e., ends of tie lines). Following Reuvers et al. [13] and Reuvers and Smolders [14], we used Dunlop's [20] method to compute the phenomenological coefficients, $L_{i j}$, using binary diffusion coefficients. The detailed procedure which involves the manipulation of binary and ternary friction coefficients are given in a previous publication [16].

\section{Experiment and computation}

\subsection{Material}

EVAL copolymer (105A) was a gift from Kuraray, Japan. It has an average molecular weight $M_{\eta}=$ $56000 \mathrm{~g} / \mathrm{mol}$ (intrinsic viscosity $=0.87 \mathrm{dl} / \mathrm{g}$ ), and an average ethylene content of $44 \mathrm{~mol} \%$. Reagent-grade DMSO (Nacalai Tesque, Kyoto, Japan) was used as the solvent for EVAL. The nonsolvent for EVAL was deionized and double-distilled water. All materials were used as received.

\subsection{Membrane preparation and characterization}

\subsubsection{Membrane preparation}

Membranes were prepared using the direct immersion-precipitation method. An appropriate amount of EVAL was dissolved in DMSO to form a $20 \mathrm{wt} \%$ homogeneous solution. This solution was dispersed uniformly on a glass plate (ca. $200 \mu \mathrm{m}$ ), and then precipitated in a nonsolvent bath to form a laminate. The compositions of the nonsolvent baths are summarized in Table 1. The nonsolvent and residual solvent were removed from this membrane by a series of washing steps. The morphology of the membrane was examined using a scanning electron microscope (SEM, Hitachi S-800) to obtain the surface and interior structures.

\subsubsection{Characterization}

The crystalline characteristics of each membrane were studied using a differential scanning calorimeter (Netsch DSC 200, Germany) over the temperature range $25-180^{\circ} \mathrm{C}$ at a heating rate of $10^{\circ} \mathrm{C} / \mathrm{min}$. Pure water flux at $25 \pm 3^{\circ} \mathrm{C}$ for each membrane was measured using a $45 \mathrm{~mm}$ diameter Stirred Ultrafiltration Cell (Amicon, Model 8050). Data were taken after the permeate flow rate had reached a stable constant value 
Table 1

Measured and calculated precipitation times for various coagulation baths

\begin{tabular}{lllll}
\hline Membrane ID & Bath DMSO (wt $\%)$ & Content (vol\%) & Measured precipitation time (s) & Calculated crossing binodal time (s) \\
\hline A & 0 & 0 & $<1$ & $<1$ \\
B & 50 & 48 & $<1$ & $<1$ \\
C & 70 & 68 & 9 & 16 \\
D & 75 & 73 & 12 & 20 \\
\hline
\end{tabular}

Dope: $20 \mathrm{wt} \%$ EVAL in DMSO.

(usually a few minutes after operation). Six transmembrane pressures, $0.2,0.3,0.4,0.5,0.7$ and $0.9 \mathrm{~kg} / \mathrm{cm}^{2}$, were employed for each membrane.

\subsection{Measurement of precipitation time}

Light transmission experiments were performed to measure the time of the onset of phase separation in the membrane solution. A collimated light beam was directed to the membrane solution immersed in a nonsolvent bath. The light transmittance was recorded with a data acquisition system. The light intensity profile was plotted as a function of time. Several of the immersion cases are given in Fig. 2. The initial precipitation time is identified as the time at which transmission intensity starts to decrease and precipitation is complete when the light transmission intensity comes to a constant minimum. The detailed experimental setup and procedures are shown in a previous publication [16].

\subsection{Diffusion path computation}

A computer code developed previously is used to compute the local concentration of each component in the membrane solution and the bath regions [16]. The membrane is divided into 32 layers. The local composition of each component in any layer of the membrane solution was assumed to be represented by a single average value. The bath was divided into 400-700 layers. The input parameters for model computation can be found in Table 2 . Thermodynamic properties (i.e., concentration dependent interaction parameters) were taken from experimental measurements [11]. Binary diffusion coefficient between solvent and nonsolvent, $D_{12}$, was calculated using the Wilke-Chang equation [21]. The average value of the two infinite dilution cases was used here. Binary mutual diffusion coefficient, $D_{23}$, was taken from the
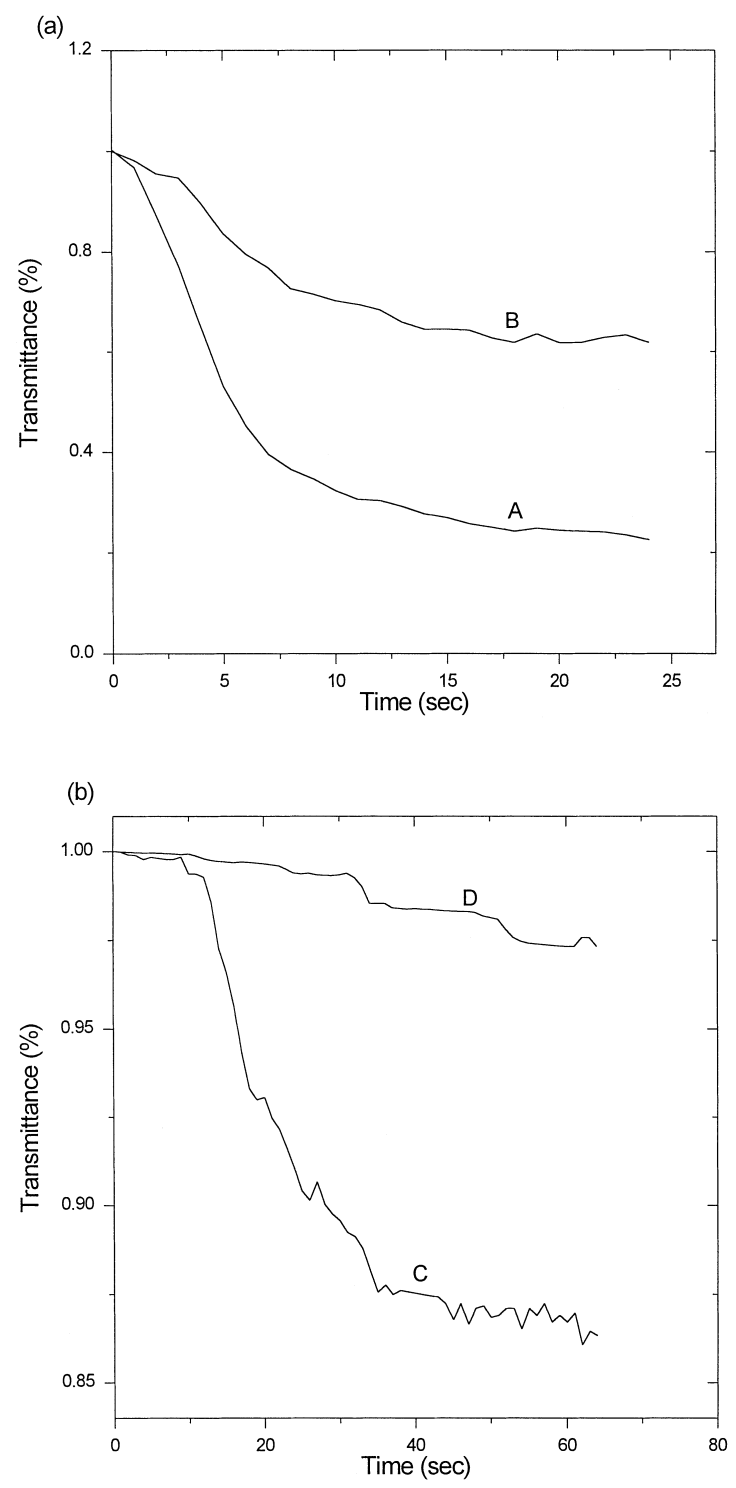

Fig. 2. Light transmission during immersion of EVAL solutions into different baths. (a) A: water bath, B: 48 vol\% DMSO in water; (b) C: 68 vol\% DMSO in water, D: 73 vol\% DMSO in water. 
Table 2

Physical parameters for diffusion trajectory calculation

\begin{tabular}{ll}
\hline & Water-DMSO-EVAL system \\
\hline$T(\mathrm{~K})$ & 298 \\
$L(\mathrm{~cm})^{\mathrm{a}}$ & 30 \\
$V_{\infty}(\mathrm{cm} / \mathrm{s})$ & 10 \\
$\nu\left(\mathrm{cm} / \mathrm{s}^{2}\right)$ & 0.01 \\
Casting thickness $(\mu \mathrm{m})$ & 200 \\
$V_{1}\left(\mathrm{~cm}^{3} / \mathrm{s}\right)$ & 18 \\
$V_{2}\left(\mathrm{~cm}^{3} / \mathrm{s}\right)$ & 71.28 \\
$V_{3}\left(\mathrm{~cm}^{3} / \mathrm{s}\right)$ & 47836 \\
$D_{12}\left(\mathrm{~cm}^{2} / \mathrm{s}\right)$ & $1.35 \times 10^{-5}$ \\
\hline
\end{tabular}

${ }^{\mathrm{a}} L$ measures the distance between the leading edge and the position of observation. When $L>30 \mathrm{~cm}$, concentration boundary layer becomes relatively flat. $D_{23}=3.404 \times 10^{-5} x^{6}-0.00011 x^{5}+$ $0.000135 x^{4}-7.445 \times 10^{-5} x^{3}+1.507 \times 10^{-5} x^{2}+9.516 \times$ $10^{-7} x-4.035 \times 10^{-8}\left(x=1-\phi_{3}\right)$.

literature average of several binary solvent-polymer systems [22] based upon the facts that these polymers have a molecular weight similar to that of EVAL and that they are all dissolved in a good solvent. To obtain friction coefficient between nonsolvent and polymer which is required for $L_{i j}$ calculations, it is assumed that the friction coefficients between polymer and low molecular species (nonsolvent and solvent) are proportional to the volume fractions of the low molecular weight species. Such an assumption has also been used elsewhere to yield satisfactory computed results [9]. Various cases corresponding to different preparation conditions are calculated. The average computation time for a set of initial condition was about $10 \mathrm{~h}$. In addition, the effect of molecular weight on membrane morphology has been reported by Matsumoto et al. [23]. However, for a thorough theoretical treatment, not only is the theory very complicated, but also the amount of experimental work needed is large. One has to determine all the relevant interaction parameters as a function of molecular weight. Therefore, the effect of molecular weight on diffusion path computation is neglected in this paper.

\section{Results and discussion}

\subsection{Diffusion trajectory and precipitation times}

In Fig. 3(a) and (b), calculated diffusion trajectories are shown for the cases of immersing a $20 \mathrm{wt} \%$ dope (a)

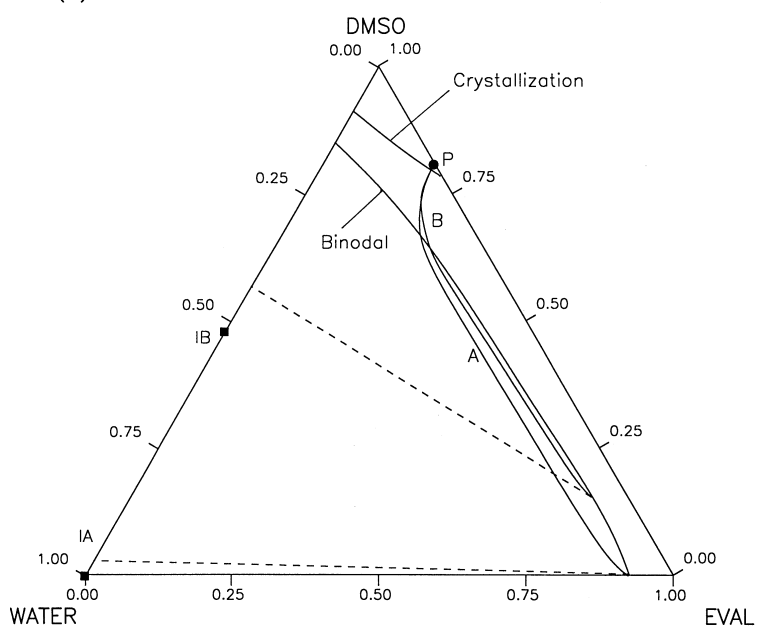

(b)

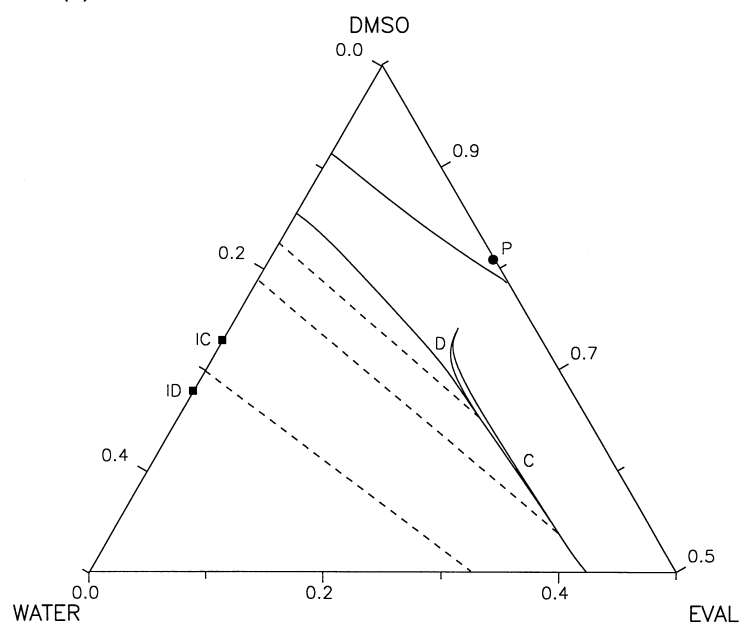

Fig. 3. Calculated diffusion trajectories at the point of precipitation for EVAL solutions immersed into various coagulation baths. Dope: point "P" (20 wt\% EVAL in DMSO); (a) A: water bath, B: 48 vol\% DMSO in water; (b) C: 68 vol\% DMSO in water, D: 73 vol\% DMSO in water. ((-- ): tie line; IA, IB, IC and ID are initial bath compositions).

solution (i.e., point $\mathrm{P}$ ) into various coagulation baths. The compositions of the baths in Fig. 3(a) are 0 and 48 vol\% DMSO in water and those in Fig. 3(b) are 68 and 73 vol\% DMSO in water. The trajectories (i.e., curves A-D in Fig. 3) represent the composition of each component in the membrane solution when the top layer just enters the binodal miscibility envelop. 
The interfacial composition of the membrane solution is located on the binodal, in accordance with the assumed equilibrium boundary condition. The time for the membrane solution to first cross the binodal is an important characteristic of the immersion process. The calculated times for various immersion conditions are summarized in Table 1. It can be seen that, for baths $\mathrm{A}$ and $\mathrm{B}$, the binodal was crossed right after immersion. Such cases are termed "instantaneous demixing" by Reuvers and Smolders [14]. The membranes formed in this type of phase separation process often have a tight skin and finger-like macrovoids. By contrast, as the coagulation bath contains a significant amount of solvent, such as the case depicted by trajectory $\mathrm{C}$ or D in Fig. 3(b), membrane solution does not enter the binodal until its bottom layer has changed the composition. This is the so-called "delayed demixing" type of precipitation [14]. If the polymer is amorphous, the formed membrane will generally have a thin and porous skin and an interior structure which is largely cellular, called sponge structure.

In Fig. 2, light transmittance profiles are shown for the immersion conditions given in Table 1. For each profile, the time at which light intensity begins to decrease is identified as the onset point of precipitation. It appears, from Fig. 2, that precipitation delayed progressively as the bath contained increasing amount of solvent. For curve D, the light intensity decreased only $3 \%$ after immersion for $60 \mathrm{~s}$ because of the similarity of refractive indices between the precipitated film and the bath. The first appreciable decrease in intensity was recognized to be at $12 \mathrm{~s}$. The measured precipitation times are compared with the calculated times for first crossing the binodal in Table 1 . These two sets of data match perfectly for the harsh baths while for the soft baths, the measured precipitation times are shorter than the calculated results. The relation between these times and the membrane morphology is further discussed below.

\subsection{Membranes prepared from harsh baths - instantaneous demixing cases}

In Fig. 4(a)-(c), morphologies of the membrane are shown for the preparation condition of immersing dope "P" in Fig. 3 into a harsh bath (pure water). This membrane has an asymmetric structure consist- ing of a thin dense skin and a thick porous support which is occupied with large finger-like macrovoids extending almost to the bottom surface. In the region among these macrovoids, as demonstrated in Fig. 4(b), a cellular morphology similar to that of an amorphous membrane is observed. These cellular pores are largely independent and are embedded in a continuous polymer matrix. Fig. 4(c) shows the top surface of the membrane. A tight skin appears to be formed. Although this surface is rough and there exist tiny crevices, it is free of submicron voids. Typical morphology characteristic of polymer crystallization (i.e., particulate, discussed previously $[5,6,10,11]$ ) is not observed in any sections of this membrane. Therefore, the phase separation process responsible for the observed immediate turbidity in light transmission measurement (curve "A", Fig. 2) is liquid-liquid demixing. The calculated diffusion trajectory for forming this membrane is shown earlier in curve "A" of Fig. 3(a), from which it can be observed that mass exchange of solvent and nonsolvent brings the membrane solution into a metastable state initially with respect to crystallization, and then with respect to liquid-liquid demixing. This suggests that although crystallization has the opportunity to take place prior to liquid-liquid demixing, precipitation in the form of nucleation and growth of liquid micelles occurs in such a rapid rate that it finishes before any induction of crystallization and a cellular morphology yields. This result illustrates that a large degree of supersaturation is not necessary and that crystallization process will dominate the resulting membrane structure. In fact, crystallization indeed occurred, but only after growth of liquid micelles had completed and the cellular morphology was fixed. Crystallization, therefore, only has a small effect on the overall morphology of the formed membrane. Evidence of crystallization during membrane formation can be found in the DSC thermograms in Fig. 5. Curve A is the melting endotherm for this membrane. It indicates a melting peak at $165^{\circ} \mathrm{C}$ and heat of fusion equal to $75 \mathrm{~J} / \mathrm{g}$. These values are close to those of pure polymer pellet specimen, and this suggests that crystallinity of the polymer is maintained in the present wet precipitation process.

The calculated polymer volume fraction corresponding to trajectory "A" in Fig. 3(a) is shown in curve "A" of Fig. 6 as a function of position along the membrane solution. The point at $x=0$ represents the 

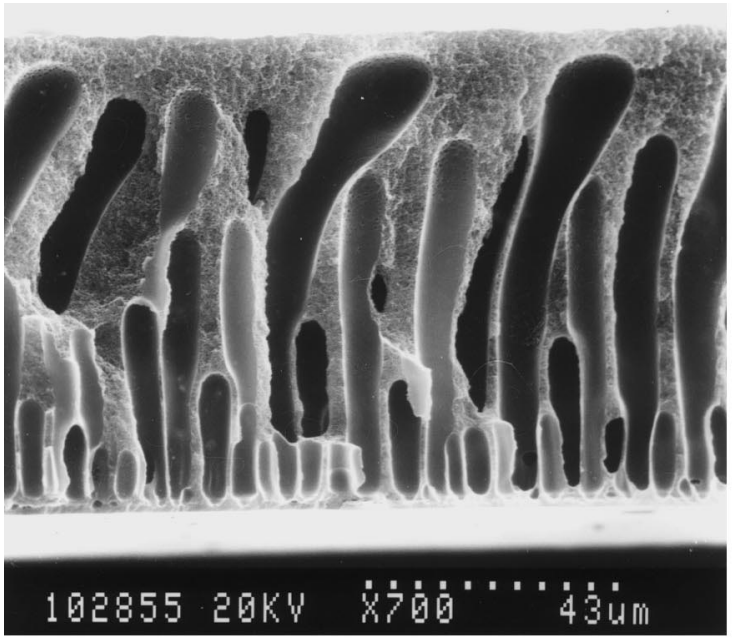

(a)

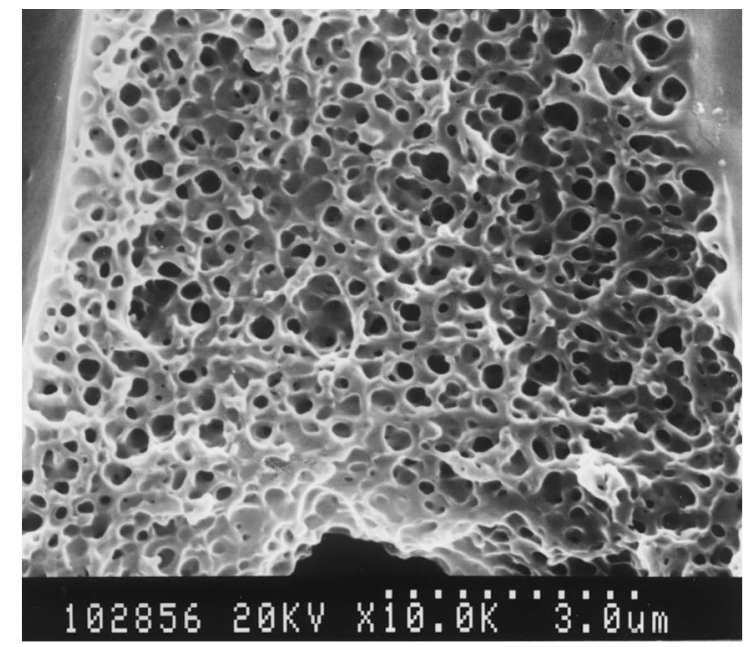

(b)

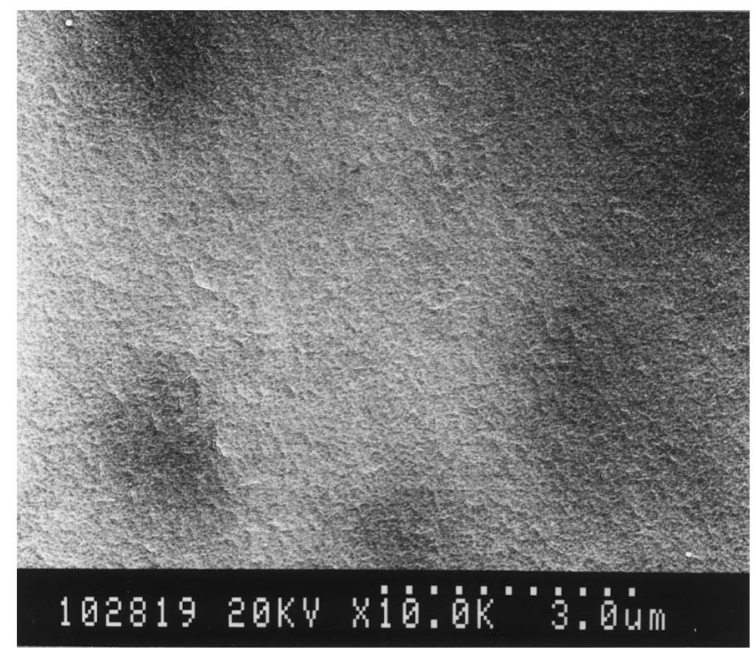

(c)

Fig. 4. SEM photomicrographs of membrane "A": (a) cross-section; (b) magnification of (a) showing the cellular pores between finger-like macrovoids; (c) top surface.

membrane-bath interface and the other end of the curve represents the bottom surface. From curve "A", a pronounced high polymer concentration $\left(\phi_{3}=0.92\right)$ resulted from the equilibrium boundary between the interface of the membrane solution and the coagulation bath was observed. Such a high polymer concentration brought rapidly this polymer-rich region into a state wherein vitrification took place and the structure froze. Hence, the skin layer was very hard and stiff so as to preclude the possibility of any microporous regions near this surface. In the membrane region underneath the skin layer, liquid-liquid phase separation occurred in terms of nucleation and growth. These liquid nuclei grew into micelles which eventually phase-inverted into cellular pores or fingerlike macrovoids. Mechanism for the formation of finger-like macrovoids is beyond the scope of the current work. However, the steep concentration gra- 


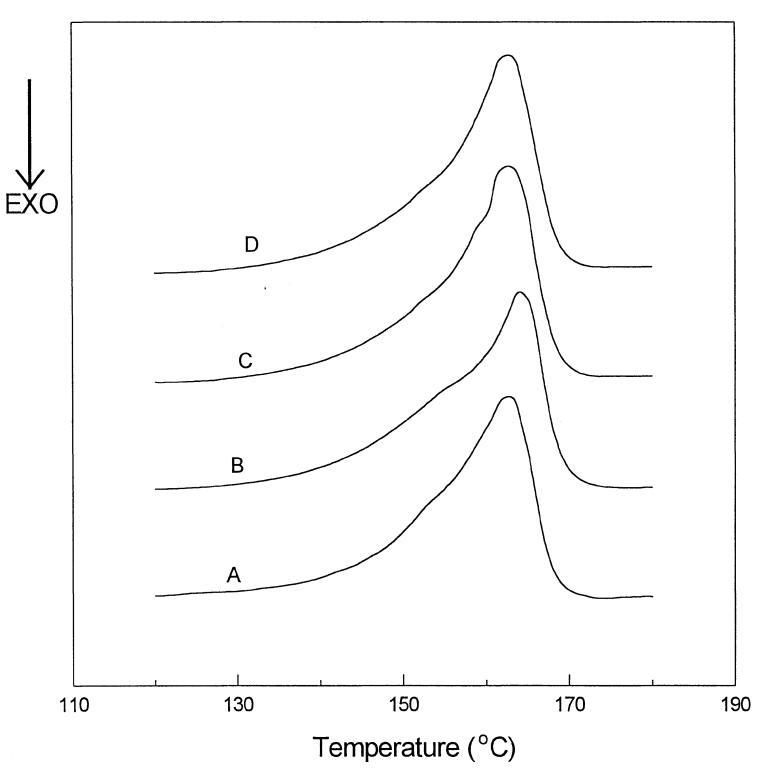

Fig. 5. DSC melting endotherms of EVAL membranes at the heating rate of $10^{\circ} \mathrm{C} / \mathrm{min} . T_{\mathrm{m}} \sim 165^{\circ} \mathrm{C}, \Delta H_{\mathrm{m}} \sim 77 \mathrm{~J} / \mathrm{g}$.

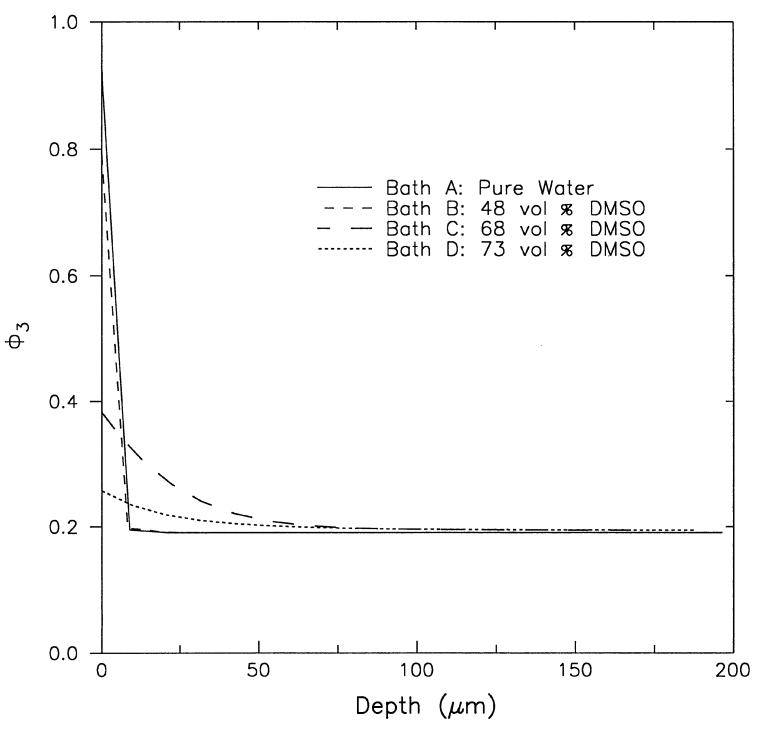

Fig. 6. Calculated polymer volume fraction profiles in membrane solution at the point of precipitation for various coagulation baths. A: water bath; B: 48 vol\% DMSO in water; C: 48 vol\% DMSO in water; D: 73 vol\% DMSO in water.

dient, as shown in Fig. 6, formed instantly on contact with the bath, indeed suggests a favorable condition for macrovoid formation [24].
Another instance of instantaneous demixing is observed when dope " $\mathrm{P}$ " is immersed in a coagulation bath containing $48 \mathrm{vol} \%$ DMSO to form membrane "B" in Table 1. The calculated diffusion trajectory (Fig. 3(a)) indicates that the binodal was crossed within $1 \mathrm{~s}$ after immersion into the coagulation bath, identical to the precipitation time obtained from light transmission measurement. Fig. 7(a)-(d) shows that membrane " $\mathrm{B}$ " is also characterized by an asymmetric cellular morphology originated from liquidliquid demixing. The macrovoids in membrane " $\mathrm{B}$ " are, however, less prevalent and the sizes of the macrovoids are significantly smaller than those in membrane " $\mathrm{A}$ ". This is consistent with the fact that macrovoids can be reduced by increasing solvent content in the bath, as was reported by many authors [25-27]. Fig. 7(c) shows the top surface of membrane "B". Compared with membrane "A" (see Fig. 4(c)), this top surface appears to be looser and there exist a number of small holes, some of which are found to be associated with the finger-like macrovoids underneath them. In Fig. 7(d), the close-up view of such a macrovoid near the top surface is shown. Strathmann [25] has proposed a surface rupture mechanism to describe the formation of this type of macrovoids. On the other hand, careful observation of the holes on the top surface shown in Fig. 7(c) indicates deposits that bulge around the hole. This suggests that the finger tip breaks up the skin as it pushes upward the swollen polymer-rich gel to form a hole on the top surface. A strong osmosis force across the micelle during macrovoid formation, as pointed out by McKelvey and Koros [28], may have taken effect in the present case. Curve "B" in Fig. 6 depicts the calculated polymer concentration at the point of precipitation for membrane " $\mathrm{B}$ ". The polymer volume in the top gel region is ca. 79 vol\% smaller than that of membrane "A". This explains why the skin of this membrane is not as rigid as that of membrane " $A$ " and why those liquid micelles under the skin can break up the gel, which dose not occur in membrane " $\mathrm{A}$ ".

\subsection{Membranes prepared from soft baths - delayed demixing cases}

As the DMSO content in the coagulation bath was raised to $68 \mathrm{vol} \%$, mass transfer slowed down and precipitation was thus delayed. Curve " $C$ " in Fig. 2 


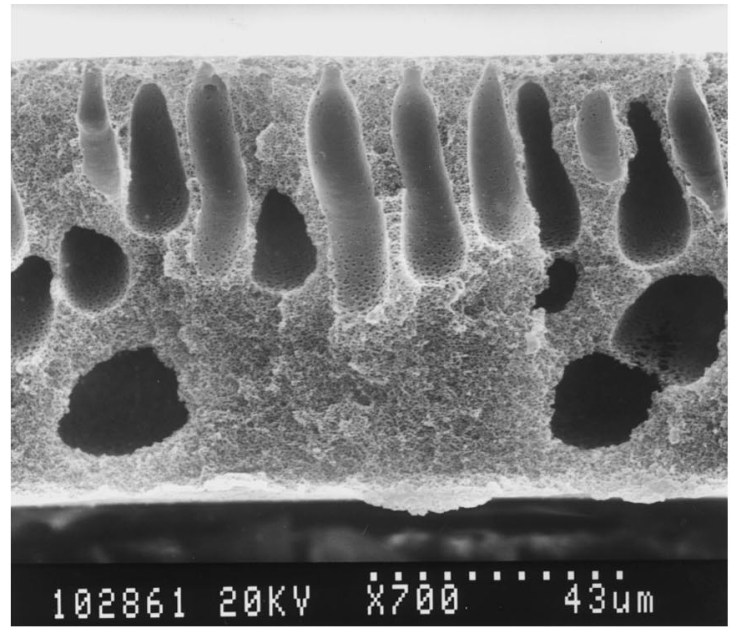

(a)

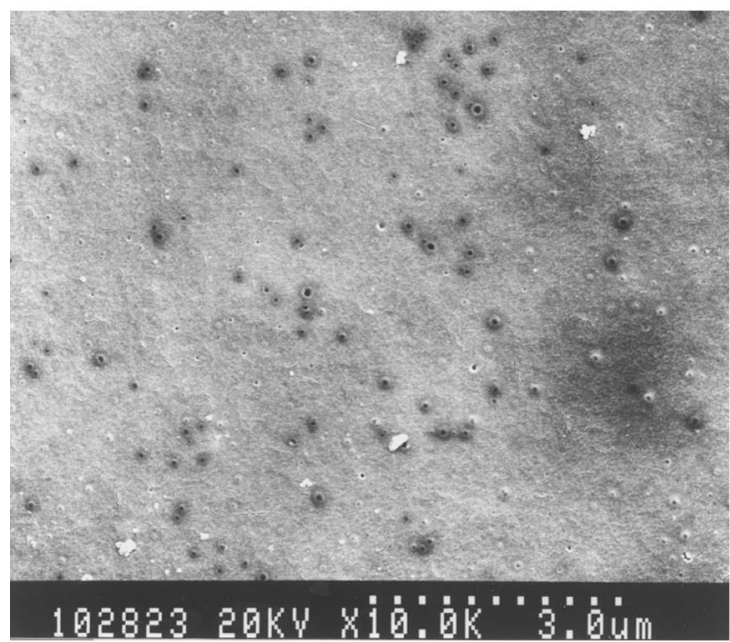

(c)

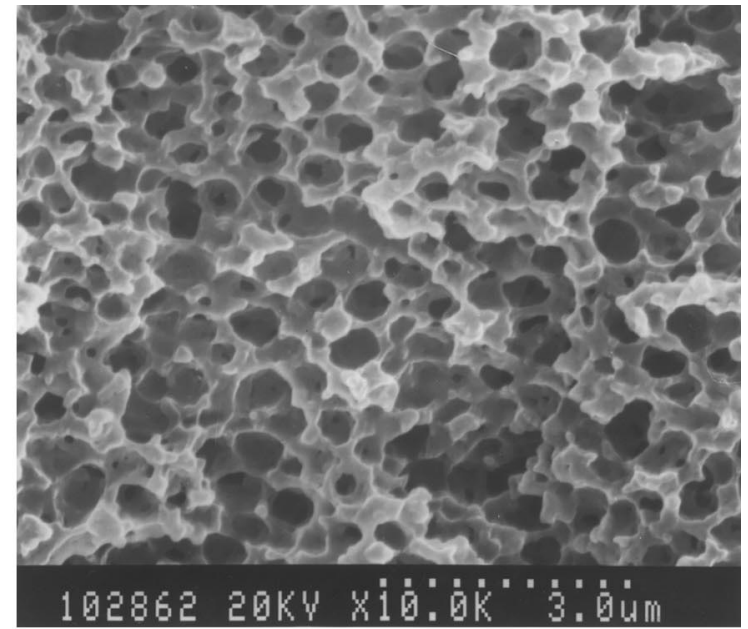

(b)

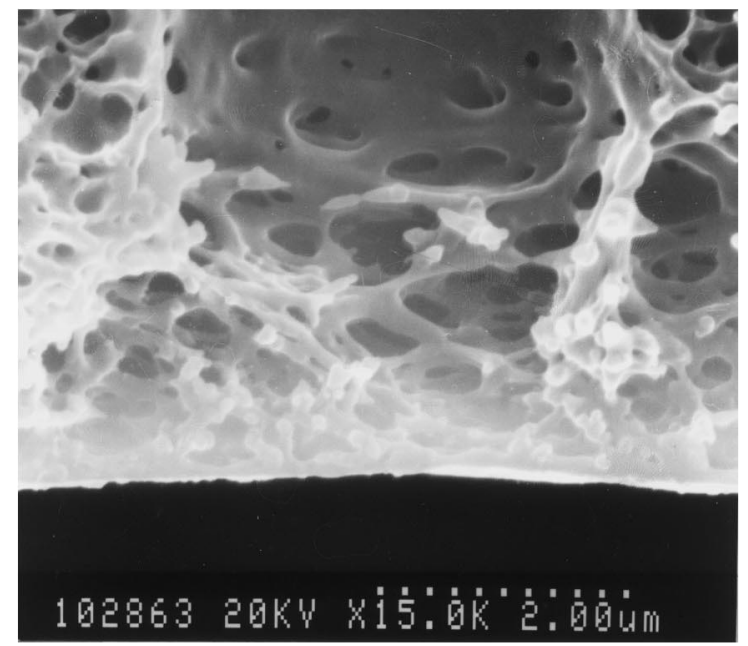

(d)

Fig. 7. SEM photomicrographs of membrane "B": (a) cross-section; (b) magnification of (a) showing the cellular pores between finger-like macrovoids; (c) top surface; (d) close-up view of a finger-like macrovoid near the top surface.

indicates a measured precipitation time of $9 \mathrm{~s}$, significantly longer than the instantaneous demixing cases. The binodal was crossed at $16 \mathrm{~s}$ after immersion, as calculated by the mass transfer model. Because of such postponement for the liquid-liquid demixing process and because the membrane solution was supersaturated with respect to crystallization, nucleation of crystallites within the membrane solution became feasible (it is, however, not certain at this point whether the observed precipitation time resulted from liquid-liquid demixing or from crystallization). The structure of this membrane (membrane " $\mathrm{C}$ " in Table 2) is shown in Fig. 8(a)-(d). It can be seen that finger-like macrovoids have completely disappeared and the membrane demonstrated a very uniform porous cross-section. The magnified edge view shown in Fig. 8(b) indicates a distorted cellular morphology in the membrane bulk, which resulted from a specific 


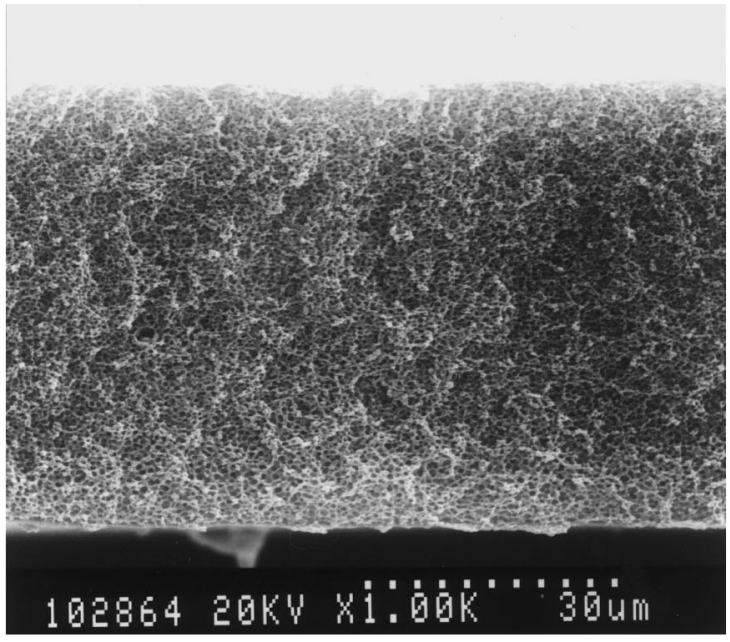

(a)

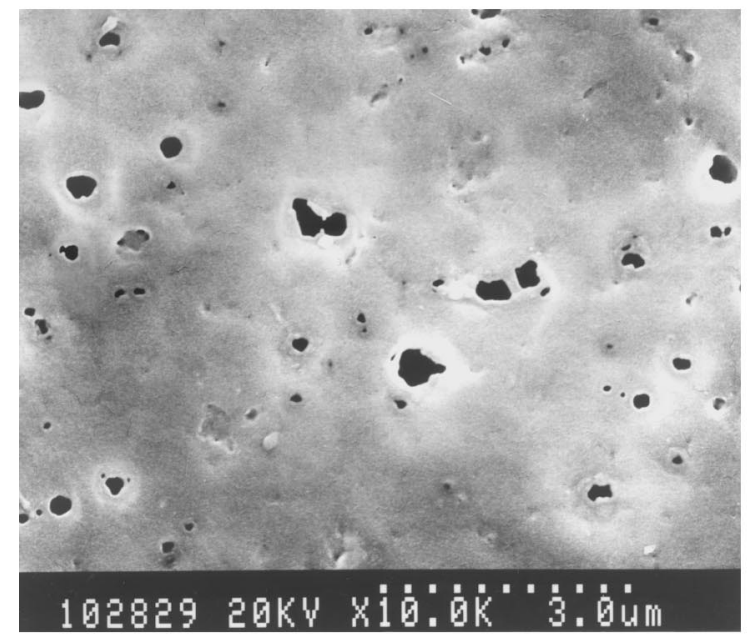

(c)

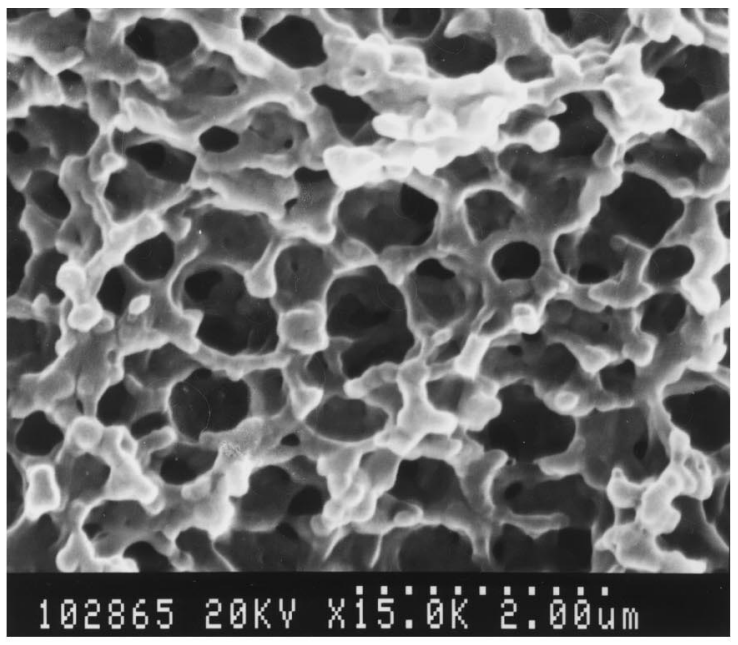

(b)

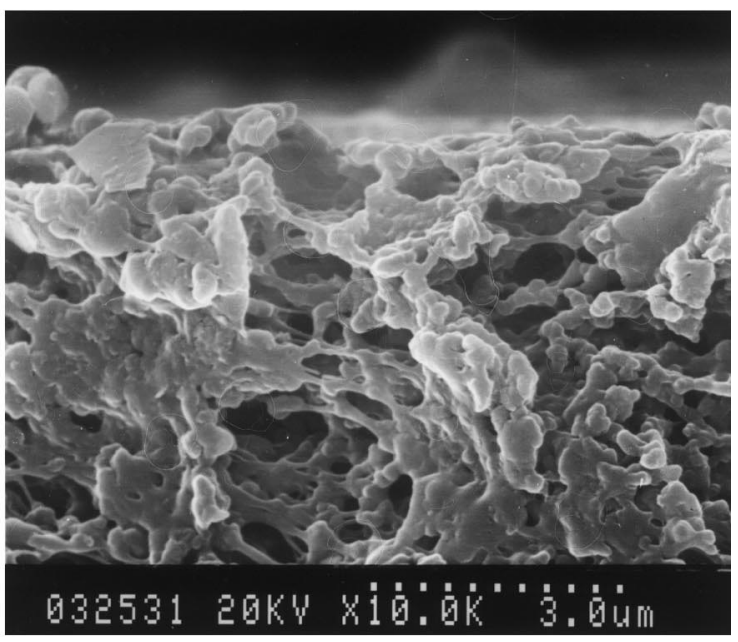

(d)

Fig. 8. SEM photomicrographs of membrane "C": (a) cross-section; (b) magnification of (a) showing a morphology mixed with cellular pores and particles; (c) top surface; (d) close-up view of the region near the top surface.

sequence of occurrence of liquid-liquid demixing and crystallization. The cellular pores, unlike those observed in membranes "A" and "B", have irregular walls that appear to be particulate. This suggests that liquid-liquid demixing might take place first and before it finished polymer crystallization started. It is also possible that liquid-liquid demixing and crystallization have occurred nearly simultaneously. In either case, the formed morphology exhibited features from both types of phase transformation, i.e., it was basically cellular but with particulate cell boundaries. As the dope was immersed into the coagulation bath, it did not enter the binodal miscibility envelop immediately. This provided an induction period for the nucleation of the crystalline particles. On the other hand, the polymer chain was not rigid enough to limit the development of crystallization process in the polymer-rich domains after the liquid-liquid demix- 
ing process had occurred. When pores were produced in the form of liquid micelles, growth of crystallites commenced in the polymer-rich cellular walls. As they grew, impurities (largely solvent and nonsolvent with composition dictated by the equilibrium crystallization line) were rejected into the micelles. As a result, the pores in the ultimate membrane were distorted and were surrounded by irregular particulate walls, and because all the pores were open and interconnected, the membrane actually developed into a "bi-continuous" morphology.

The calculated polymer concentration in the membrane solution at the outset of precipitation for membrane " $C$ " is shown in curve " $C$ " of Fig. 6. Rather than a sharp increase in polymer concentration near the top surface that happens in membranes " $A$ " and "B", curve " $C$ " shows a steady and small decrease of $\phi_{3}$ from 0.38 at the interface to 0.19 in the bulk. Because of such small difference in $\phi_{3}$ across the membrane solution, the porous structure is expected to be uniform and this is confirmed by the SEM shown in Fig. 8(a). The top surface of this membrane is shown in Fig. 8(c), from which it can be seen that pores become significant in this top surface. These pores are separated and randomly distributed. Some of them appear to be connected with the pores in the membrane bulk (also indicated in Fig. 8(d)). The sizes of these pores are several times larger than those at top surface of membrane " $\mathrm{B}$ ". This is consistent with the calculated interfacial polymer concentrations for these cases shown in Fig. $6\left(\phi_{3}=0.38\right.$ and 0.7 , respectively, for curves "C" and "B"). Since curve " $C$ " has an interfacial polymer concentration significantly lower than that of curve " $B$ ", the top gel layer of membrane " $C$ " should be weaker and is more likely to be broken up by the growing liquid micelles in this layer.

As dope solution "P" was immersed in a very soft coagulation bath, i.e. bath "D" containing 73 vol\% DMSO, liquid-liquid demixing process was sufficiently suppressed and crystallization dominated the precipitation process. The formed membrane exhibited a typical particulate morphology characteristic of EVAL crystallization as shown in the membrane cross-sectional region. SEM photomicrographs of this membrane are shown in Fig. 9(a)-(c). The light transmission experiment indicates a delayed precipitation occurring at $12 \mathrm{~s}$ (see Table 1 or Fig. 2), somewhat shorter than the calculated time for the top layer to cross the binodal. At the time of commencement of phase transformation, the polymer volume fraction in the membrane bulk is essentially constant, as shown in curve "D" of Fig. 6, which suggests a uniform crosssection for the formed membrane. This is confirmed by the SEM shown in Fig. 9(a). The magnified edge view of this membrane is shown in Fig. 9(b). It can be seen that globular EVAL particles (ca. $0.2 \mu \mathrm{m}$ diameter) join each other to form a homogeneous continuous mat with considerable mechanical cohesiveness. There is little evidence of a regular cellular pore structure that is initiated by the process of liquid-liquid demixing. Because crystallization is the dominant phase transformation, it initiates the precipitation process and is responsible for the observed turbidity in the light transmittance measurement. As shown in Fig. 6, the interfacial polymer volume fraction increases only slightly from 0.19 to 0.26 . This results in an extremely soft polymer gel zone near the membrane-bath boundary, which is easily broken up during phase inversion of micelles in this zone. Consequently, this membrane does not have a continuous skin, as is illustrated by the perforated top surface shown in Fig. 9(c).

The DSC thermograms of the EVAL membranes precipitated from different bath conditions are shown in Fig. 5. It appears that all membranes have a melting peak at ca. $163^{\circ} \mathrm{C}$ and an enthalpy of fusion of ca. $77 \mathrm{~J} /$ $\mathrm{g}$ close to that of a melt-crystallized polymer. This implies that although liquid-liquid demixing may initiate the precipitation process and dominate the formed morphology (e.g., cellular pores in membranes "A" and "B"), crystallization actually occurs in the phase separated polymer-rich domain in the nascent membrane. The nucleation of EVAL polymer crystallites depends on the degree of supersaturation of the local composition with respect to the crystallization line. After liquid-liquid demixing has occurred, the polymer-rich phase, whose composition is dictated by the equilibrium binodal, falls deeply into the crystallization region (for the present system, the tie lines of the binodal have negative slopes as shown in Fig. 3). Crystallization will take place in this highly supersaturated polymer-rich phase. However, because the growth of liquid pores have completed, the structure has largely been fixed. Crystallization affects the ultimate membrane morphology only to an insignificant extent (see Figs. 4 and 7). 


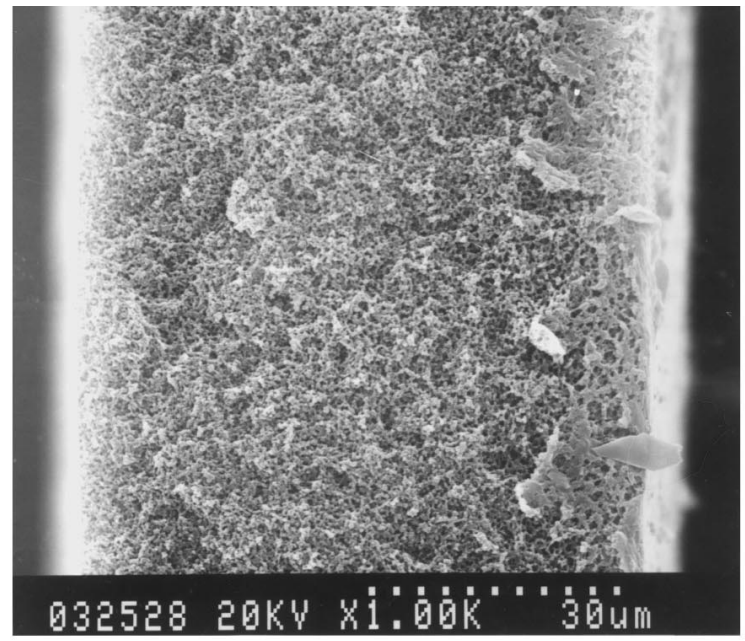

(a)

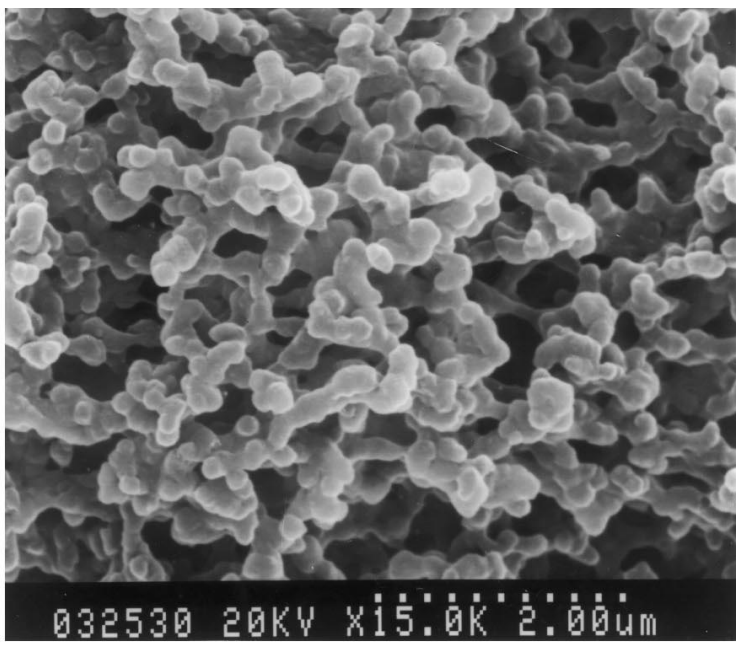

(b)

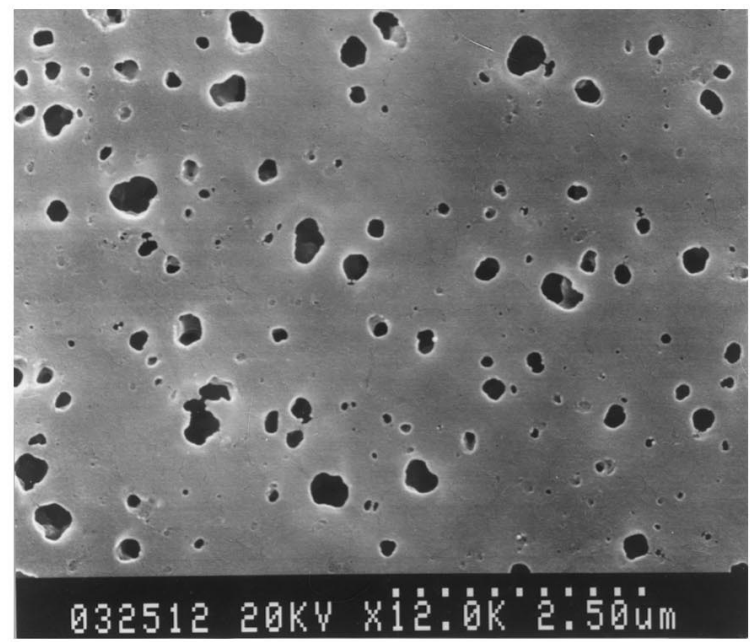

(c)

Fig. 9. SEM photomicrographs of membrane "D": (a) cross-section; (b) magnification of (a) showing the typical particulate morphology; (c) top surface.

\subsection{Pure water flux and the membrane morphology}

The pure water fluxes for various EVAL membranes are presented as a function of transmembrane pressures in Fig. 10. As expected, the water flux increases corresponding to the pressure for all membranes. The water flux data actually reflect the morphology of the membrane. Both membranes "A" and "B" have an asymmetric cellular structure. Their water permeabil- ities are very low due to the tight skin and the largely closed pores. Although membrane " $\mathrm{A}$ " has more prevalent and larger finger-like macrovoids than membrane " $\mathrm{B}$ ", its skin is tighter and thus has a slightly lower water flux than membrane "B". The water permeability of membrane $\mathrm{C}$ is much higher than both membranes " $\mathrm{A}$ " and " $\mathrm{B}$ " because its cellular pores are largely open (resulting from irregular particulate cell boundaries) and its top surface has considerably 


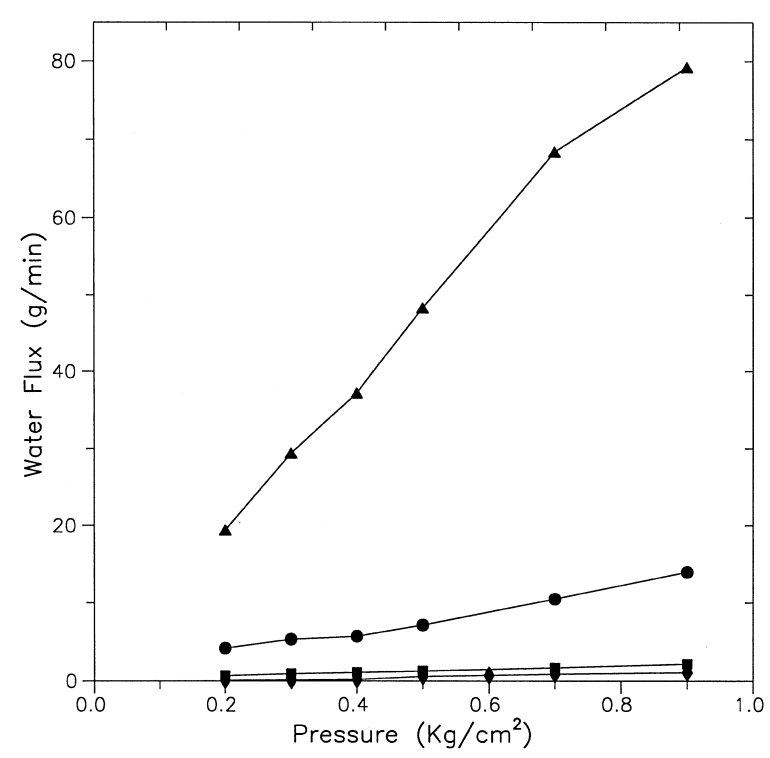

Fig. 10. The pure water fluxes of EVAL membranes: membrane "A"; ( $\mathbf{\square})$ membrane "B"; $(\mathbf{O})$ membrane "C"; ( $)$ membrane "D".

more pores. The water flux of membrane " $\mathrm{D}$ " is the highest of all membranes. At the pressure of $0.9 \mathrm{~kg} /$ $\mathrm{cm}^{2}$, the water flux reaches ca. $80 \mathrm{~g} / \mathrm{min}$ per unit area, a value typical of microfiltration applications. Since membrane " $D$ " is skinless and has a uniform particulate morphology in which all voids are interconnected, water molecules can, therefore, flow through the continuous void channels with rather low resistance.

\section{Conclusion}

EVAL membranes were prepared by isothermal immersion-precipitation from water-DMSO solutions. Depending upon the sequence of the precipitation processes, the formed membranes may exhibit cellular (characteristic of liquid-liquid demixing) and particulate (characteristic of crystallization) structures. If the bath is soft, crystallization may precede liquid-liquid demixing and a particulate bi-continuous morphology will form. Calculations of the concentration profiles indicate the formation of a thin soft gel near the top surface during precipitation. This leads to the observed unskinned top surface in the formed membrane. For the case of immersing in a harsh bath, liquid-liquid phase separation dominates and an asymmetric cellular morphology is formed. Since in this case there is a sharp increase of polymer concentration near the boundary region between membrane and bath, a dense gel at the top surface is formed, which eventually develops into the tight skin in the final structure. The permeability of EVAL membranes appears to depend on the microporosity and interconnectivity of the pores. Water flux data point out that skinless bi-continuous membranes prepared in soft baths are much more permeable than the skinned membranes precipitated from harsh baths.

\section{Acknowledgements}

The authors thank the National Science Council of Taiwan, Republic of China, for the financial support, Grant \#:NSC 85-2216-E-032-001.

\section{References}

[1] K. Maier, E. Scheuermann, Über die Bildungsweise teildurchlässiger Membranen, Kolloid Z. 171 (1960) 122.

[2] R.E. Kesting, Synthetic Polymeric Membranes, Wiley, New York, 1985.

[3] M. Mulder, Basic Principles of Membrane Technology, Kluwer Academic Publishers, Dordrecht, 1991.

[4] L.P. Cheng, A.W. Dwan, C.C. Gryte, Isothermal phase behavior of nylon-6, -66 , and -610 polyamides in formic acid-water systems, J. Polym. Sci., Polym. Phys. Ed. 32 (1994) 1183.

[5] L.P. Cheng, A.W. Dwan, C.C. Gryte, Membrane formation by isothermal precipitation in polyamide-formic acid-water systems. I. Description of membrane morphology, J. Polym. Sci., Polym. Phys. 33 (1995) 211.

[6] A.M.W. Bulte, B. Folkers, M.H.V. Mulder, C.A. Smolders, Membranes of semicrystalline aliphatic polyamide nylon 4,6: Formation by diffusion-induced phase separation, J. Appl. Polym. Sci. 50 (1993) 13.

[7] A.M.W. Bulte, M.H.V. Mulder, C.A. Smolders, H. Strathmann, Diffusion induced phase separation with crystallizable nylons. I. Mass transfer processes for nylon 4,6, J. Membr. Sci. 121 (1996) 37.

[8] B. Wunderlich, Macromolecular Physics, Academic Press, New York, 1973.

[9] L.P. Cheng, A.W. Dwan, C.C. Gryte, Membrane formation by isothermal precipitation in polyamide-formic acid-water systems. II. Precipitation dynamics, J. Polym. Sci., Polym. Phys. 33 (1995) 223. 
[10] L.P. Cheng, H.Y. Lin, L.W. Chen, T.H. Young, Solute rejection of dextran by EVAL membranes with asymmetric and particulate morphologies, Polymer 39 (1998) 2135.

[11] T.H. Young, J.Y. Lai, W.M. You, L.P. Cheng, Equilibrium phase behavior of the membrane forming water-DMSOEVAL copolymer system, J. Membr. Sci. 128 (1997) 55.

[12] C. Cohen, G.B. Tanny, S. Prager, Diffusion controlled formation of porous structures in ternary polymer solutions, J. Polym. Sci., Polym. Phys. Ed. 17 (1979) 477.

[13] A.J. Reuvers, J.W.A. van den Berg, C.A. Smolders, Formation of membranes by means of immersion precipitation. Part I. A model to describe mass transfer during immersion precipitation, J. Membr. Sci. 34 (1987) 45.

[14] A.J. Reuvers, C.A. Smolders, Formation of membranes by means of immersion precipitation. Part II. The mechanism of formation of membranes prepared from the system CA/ acetone/water, J. Membr. Sci. 34 (1987) 67.

[15] C.S. Tsay, A.J. McHugh, Mass transfer modeling of asymmetric membrane formation by phase inversion, J. Polym. Sci., Polym. Phys. Ed. 28 (1990) 1327.

[16] L.P. Cheng, Y.S. Soh, A.W. Dwan, C.C. Gryte, An improved model for mass transfer during the formation of polymeric membranes by the immersion-precipitation process, J. Polym. Sci., Polym. Phys. Ed. 32 (1994) 1413.

[17] J.S. Vrentas, J.L. Duda, Prediction of diffusion coefficients for polymer-solvent systems, AIChE J. 28 (1982) 279.

[18] D.D. Fitts, Nonequilibrium Thermodynamics, McGraw-Hill, New York, 1962.
[19] L.P. Cheng, Doctoral Dissertation, Columbia University, New York, 1993.

[20] P.J. Dunlop, Frictional coefficients for binary and ternary isothermal diffusion, J. Phys. Chem. 68 (1964) 26.

[21] R.C. Reid, J.M. Prausnitz, T.K. Sherwood, The Properties of Gases and Liquids, 3rd ed., McGraw-Hill, New York, 1997.

[22] L.P. Cheng, A.W. Dwan, C.C. Gryte, Measurements of mutual diffusivities in concentrated solutions of membrane-forming polyamides and cellulose acetate, J. Appl. Polym. Sci. 57 (1995) 563.

[23] T. Matsumoto, K. Nakamae, T. Ochiumi, S. Horie, Effect of molecular weight of ethylene-vinyl alcohol copolymer on membrane properties, J. Membr. Sci. 9 (1981) 109.

[24] A.J. McHugh, C.S. Tsay, Dynamics of the phase inversion process, J. Appl. Polym. Sci. 46 (1992) 2011.

[25] H. Strathmann, Production of microprobes media by phase inversion process, in: D.R. Lloyd (Ed.), Material Science of Synthetic Membrane, ACS Symposium Series, 1985.

[26] C.A. Smolders, A.J. Reuvers, R.M. Boom, I.M. Wienk, Microstructures in phase-inversion membranes. Part 1. Formation of macrovoids, J. Membr. Sci. 73 (1992) 259.

[27] T.H. Young, L.W. Chen, A two step mechanism of diffusion controlled ethylene vinyl alcohol membrane formation, J. Membr. Sci. 57 (1991) 69.

[28] S.A. McKelvey, W.J. Koros, Phase separation, vitrification, and manifestation of macrovoids in polymeric asymmetric membranes, J. Membr. Sci. 112 (1996) 29. 